\title{
Diagnosis and management of 528 abdominal aortic aneurysms
}

\author{
J W L FIELDING, J BLACK, F ASHTON, G SLANEY, D J CAMPBELL
}

\begin{abstract}
Between 1960 and 1979528 patients with abdominal aortic aneurysms presented to the university department of surgery. Of these, $222(42 \%)$ were elective cases, 72 acute (14\%), 174 had ruptured $(33 \%)$, and four had had a spontaneous aortoduodenal fistula (1\%). In all these patients resections were undertaken, but in another 56 patients $(11 \%)$ the aneurysm was not resected. A review of these cases showed that $91 \%$ had symptoms at their first presentation; abdominal pain and backache being most common. The diagnosis could be established in $91 \%$ by the presence of a pulsatile abdominal mass on clinical examination. The operative mortality for elective resection was $8 \%$, for acute $19 \%$, for ruptured cases $42 \%$, and for spontaneous aortoduodenal fistula $50 \%$. After successful resection the overall five-year survival was $65 \%$ by the life table method, and there was no significant difference between elective, acute, and ruptured cases.

This five-year survival after resection compares favourably with the expected $76 \%$ survival of a similar normal population, and was considerably better than
\end{abstract}

\footnotetext{
Department of Surgery, Queen Elizabeth Hospital, Queen Elizabeth Medical Centre, Edgbaston, Birmingham B15 2TH

J W L FIELDING, FRCs, lecturer in surgery, University of Birmingham

J BLACK, MD, FRCS, lecturer in surgery (present appointment: consultant surgeon, Worcester Royal Infirmary)

F ASHTON, CHM, FRCS, professor of surgery, University of Birmingham

G SLANEY, CHM, FRCs, professor of surgery, University of Birmingham

D J CAMPBELL, FRCS, senior registrar (present appointment: consultant surgeon, Selly Oak Hospital, Birmingham)
}

that for the conservatively treated patients. As most cases have symptoms, and diagnosis may be established easily by routine physical examination in $91 \%$, the prognosis for this condition could be considerably improved by increased awareness of its existence and early referral for treatment as an elective surgical procedure.

\section{Introduction}

Infrarenal aortic aneurysms produce symptoms, most commonly abdominal pain and backache, and the diagnosis may easily be established by abdominal examination. If referred for elective resection the operative mortality is low and the five-year survival good. Should the aneurysm not be recognised at this stage, however, and it subsequently ruptures, the prognosis is significantly worse, which is solely attributable to the high operative mortality associated with emergency resections.

Aneurysms are a sign of atheromatous disease, which is the most common cause of death in the United Kingdom. Abdominal aortic aneurysms are present in $1-4 \%$ of the population over the age of $50,1^{2}$ and, of patients dying with an unresected ruptured abdominal aortic aneurysm, the diagnosis is completely missed in $52 \%{ }^{3}$ During the past 30 years there have been striking advances in reconstructive arterial surgery, owing to improved surgical and anaesthetic techniques and the development of efficient arterial prostheses. Among these advances has been the excision and replacement of aortic aneurysms, rupture of which had been invariably fatal.

We report a series of 528 patients with infrarenal aortic aneurysms and evaluate their clinical presentation, methods of diagnosis, and the results of surgical excision with reference to postoperative mortality and long-term survival. This has shown features that have important implications for management, particularly in diagnosis and treatment. 


\section{Materials and methods}

We have reviewed all the patients presenting with infrarenal aortic aneurysms in the university department of surgery from 1960 to 1979 to establish the clinical symptoms and physical signs associated with this condition and to evaluate the methods of diagnosis.

The surgical management has been resection of the aneurysm and its replacement with a synthetic prosthesis, initially Teflon and subsequently Dacron, together with antibiotic prophylaxis. The resections were denoted as elective when a planned procedure was performed, or acute when rapid expansion in the size of aneurysm or the sudden onset of symptoms with a tender aneurysm on palpation necessitated urgent operation, but at which, nevertheless, the aneurysm was intact. Aneurysmal rupture denotes leakage associated with a frank retroperitoneal or intraperitoneal haemorrhage. Mortality has been defined as postoperative when death occurred during or subsequent to resection at any time during the initial hospital admission, but the figures for the usual conventional 30-day period are also reported. Long-term survival in all patients available for follow-up at the end of 1978 was established by follow-up in outpatient clinics or by a questionnaire sent to the family doctor. Analysis of long-term survival has been by the life table method."

\section{Results}

In the 20-year period $1960-79,528$ patients with a diagnosis of infrarenal aortic aneurysm were seen in the department of surgery. Of the 472 patients who had a resection, $222(42.1 \%)$ were elective, 72 acute $(13.6 \%), 174$ had ruptured $(32.9 \%)$, and four had developed a spontaneous aortoduodenal fistula $(0.8 \%)$. In the remaining 56 patients the aneurysm was not resected at the initial referral for reasons detailed later (table $I$ ). The number of cases treated surgically has increased in each subsequent quinquenium except the last, but the proportion of elective, acute, and ruptured cases has not changed significantly over the 20 years under review (table II).
TABLE III-Mean age at presentation

TABLE II-Incidence of resectable aneurysms per quinquenia. (Percentages in parentheses)

\begin{tabular}{lccccc}
\hline Category & $\begin{array}{c}1960-4 \\
(\mathrm{n}=61)\end{array}$ & $\begin{array}{c}1965-9 \\
(\mathrm{n}=109)\end{array}$ & $\begin{array}{c}1970-4 \\
(\mathrm{n}=141)\end{array}$ & $\begin{array}{c}1975-9 \\
(\mathrm{n}=157)\end{array}$ & Total \\
\hline Elective & $34(55 \cdot 7)$ & $50(45 \cdot 9)$ & $67(47 \cdot 5)$ & $71(45 \cdot 2)$ & $222(47 \cdot 4)$ \\
Acute & $8(13 \cdot 1)$ & $14(12 \cdot 8)$ & $23(16 \cdot 3)$ & $27(17 \cdot 2)$ & $72(15 \cdot 4)$ \\
Ruptures* & $19(31 \cdot 2)$ & $45(41 \cdot 3)$ & $51(36 \cdot 2)$ & $59(37 \cdot 6)$ & $174(37 \cdot 2)$ \\
& & & & & \\
\hline
\end{tabular}

*Excludes four cases of aortoduodenal fistula.

\section{CLINICAL PRESENTATION AND DIAGNOSIS}

There were 462 men and 66 women, whose mean age was virtually the same in all three groups studied (table III). Abdominal pain was the most common presenting symptom, occurring in $47.7 \%$ of routine cases and increasing to $83.6 \%$ when rupture had taken place. Backache was also frequent, being present in $35.3 \%$ of elective, $67.1 \%$ of acute, and $51.4 \%$ ruptured cases (table IV). Only 48 of the 528 patients were completely asymptomatic with an incidental aneurysm found at examination for another complaint. Of 151 patients with ruptured aneurysms, 107 showed clinical features of shock, and in 75 of these the blood pressure was below $100 \mathrm{~mm} \mathrm{Hg}$. Nine patients had had a malignant condition treated and a further 11 on admission for their aneurysmectomy were found to have cancer.

\begin{tabular}{lccc}
\hline & Elective & Acute & Ruptures \\
\hline Survived & $68 \cdot 5$ & $68 \cdot 0$ & $67 \cdot 4$ \\
No & 204 & 58 & 100 \\
Range & $37-84$ & $31-85$ & $41-85$ \\
Died & 67.5 & $65 \cdot 5$ & $68 \cdot 4$ \\
No & 18 & 14 & $73^{*}$ \\
Range & $55-81$ & $57-81$ & $49-84$ \\
\hline
\end{tabular}

* One not known.

TABLE IV-Symptoms at presentation. (Percentages in parentheses)

\begin{tabular}{|c|c|c|c|c|c|c|}
\hline & & & & Elective & Acute & Rupture $†$ \\
\hline $\begin{array}{l}\text { Total .. } \\
\text { Not known } \\
\text { Total known } \\
\text { One symptom } \\
\text { Pain } \\
\text { Backache } \\
\text { Mass noticed } \\
\text { Embolic symp } \\
\text { Other*.. } \\
\text { None . . } \\
\text { Claudication }\end{array}$ & $\begin{array}{l}\ldots \\
\therefore \\
\therefore \\
\ldots \\
\text { by pat } \\
\text { ptoms } \\
\ldots \\
\ldots \\
\ldots\end{array}$ & $\begin{array}{c}\ldots \\
\cdots \\
\cdots \\
\cdots \\
\cdots \\
\ldots \\
\ldots \\
\ldots \\
\ldots\end{array}$ & $\begin{array}{l}\ldots \\
\ldots \\
\ldots \\
\cdots \\
\cdots \\
\cdots \\
\ldots \\
\ldots\end{array}$ & $\begin{array}{l}222 \\
4 \\
218 \\
107(49 \cdot 1) \\
104(47 \cdot 7) \\
77(35 \cdot 3) \\
37(17 \cdot 0) \\
5(2 \cdot 3) \\
48(22 \cdot 0) \\
33(15 \cdot 1) \\
44(20 \cdot 2)\end{array}$ & $\begin{array}{l}72 \\
2 \\
70 \\
29(41 \cdot 4) \\
53(75 \cdot 7) \\
47(67 \cdot 1) \\
5(7 \cdot 1) \\
2(2 \cdot 9) \\
20(28 \cdot 5) \\
0 \\
10(14 \cdot 3)\end{array}$ & $\begin{array}{c}174 \\
28 \\
146 \\
52(35 \cdot 6) \\
122(83 \cdot 6) \\
75(51 \cdot 4) \\
5(3 \cdot 4) \\
2(1 \cdot 4) \\
66(45 \cdot 2) \\
0 \\
8(5 \cdot 5)\end{array}$ \\
\hline
\end{tabular}

*Included symptoms such as weight loss, vomiting, and change in bowel habit that were present in addition to symptoms listed in this table.

tExcludes four cases of aortoduodenal fistula.

In 392 of 431 patients the diagnosis was established by the presence of a pulsatile abdominal mass on physical examination. Plain abdominal radiography performed in $\mathbf{1 7 0}$ of the acute or elective cases confirmed the diagnosis in $129(75.9 \%)$. Only 22 patients had aortograms, which confirmed the diagnosis in 13, but in the remainder the findings were either equivocal or misleading. Five patients known to have abdominal aortic aneurysms presented as emergencies with suspected rupture but at laparotomy were found to have gangrenous bowel, the aneurysm being intact. Thus $2.8 \%$ of ruptured aneurysms were diagnosed incorrectly. A further patient was also operated on for an ischaemic bowel in the presence of a known aneurysm.

All this indicates that an abdominal aortic aneurysm is usually easily detectable on palpation and is a common cause of abdominal discomfort and backache in the middle-aged and elderly patient.

\section{Treatment}

Altogether 486 patients (92\%) underwent laparotomies for infrarenal abdominal aortic aneurysms (table I). At operation in five of these, however, the procedure was abandoned because of extension above the renal arteries. Two other patients were found to have carcinomatosis, another had extensive retroperitoneal fibrosis precluding resection of the aneurysm, and in six the symptoms were due to ischaemic gut.

Forty-two patients with a known aneurysm did not have an operadied on the way to theatre or in the anaesthetic room, one resolutely refused operation, one died undiagnosed after an emergency operation for a bleeding gastric ulcer, one had extension above the renal arteries, and the remaining patient, aged 80 , had chronic obstructive airways disease. Ten patients had advanced distal occlusive peripheral vascular disease and presented with severe claudication or rest pain and gangrene, or both; of these, one had a $3 \mathrm{~cm}$ aneurysm and the remaining nine had asymptomatic aneurysms. Eleven patients were considered unfit on medical grounds and a further six were asymptomatic. Of these 27 patients having no operation because of severe occlusive disease, lack of symptoms, or medical unfitness, five subsequently died of aneurysmal rupture $(18.5 \%)$, four of myocardial infarcts, two of tion (table I). Eight of these had ruptured aneurysms, four of whom 
BRITISH MEDICAL JOURNAL VOLUME $283 \quad 1$ AUGUST 1981

cerebrovascular accidents, two of cancer, one of respiratory failure, while 10 remained alive and three were lost to follow-up. The remaining seven patients admitted for aneurysmectomy did not have a resection because a malignant condition was detected during preoperative assessment; five had bronchial carcinomas, one a gastric carcinoma, and one carcinoma of the bladder.

\section{RESULTS OF TREATMENT}

\section{Postoperative mortality}

Of the 472 patients who had their infrarenal abdominal aortic aneurysms resected, 108 died postoperatively $(22.9 \%), 101$ within 30 days of surgery $(21.4 \%)$, and seven after this time from operative complications $(1.5 \%)$. The overall operative mortality for patients treated by elective surgery was $8.1 \%$. This figure includes all late deaths that were related to surgery, but for patients dying within the conventional 30-day period the operative mortality was $6.3 \%$. The overall operative mortality for acute cases was $19.4 \%$ and $18.1 \%$ within 30 days. The corresponding figures for ruptured cases were $42.5 \%$ and $41.3 \%$. The operative mortality for the four cases of spontaneous aortoduodenal fistulae was $50 \%$. These differences have been analysed using Chi-squared test with Yates's correction and are statistically significant (table V).

TABLE $\mathrm{v}-$ Postoperative mortality

\begin{tabular}{lcccc}
\hline & Elective & Acute & Rupture & $\begin{array}{c}\text { Aortoduodenal } \\
\text { fistula }\end{array}$ \\
\hline De aths & & & & \\
30 days & 14 & 13 & 72 & 2 \\
30 days & 4 & 1 & 2 & 0 \\
Total deaths & 18 & 14 & 74 & 2 \\
Total patients & 222 & 72 & 174 & 4 \\
Mortaiity $(\circ .)$. & $8 \cdot 1$ & $19 \cdot 4$ & $42 \cdot 5$ & 50 \\
\hline
\end{tabular}

Significance of difference using $\chi^{2}$ test with Yates's correction: elective $v$ rupture $\chi^{2}=62 \cdot 88, p-0.0005 ;$ elective $v$ acute $\chi^{2}=6 \cdot 08, p-0.02 ;$ acute $v$ rupture $\chi^{2}=10 \cdot 28$,
$\mathrm{p}=0.0005$.

The mean age of patients presenting for elective or emergency surgery and those who survived or died after operation was very similar (table III). The postoperative mortality of elective cases was $6.5 \%$ in the 153 patients under 70 and $11.6 \%$ in the 69 over 70 , but the difference is not statistically significant $\left(\chi^{2}=1.02\right)$. In the acute cases the postoperative mortality was $25.5 \%$ in 47 patients under 70 and $8 \%$ in 25 patients above, but again the difference is not statistically significant $\left(\chi^{2}=2 \cdot 18\right)$. In the patients presenting with a ruptured aneurysm the influence of age on mortality was also not statistically significant, being $38.2 \%$ in the 102 patients under 70 and $47.9 \%$ in the 71 patients over $70\left(\chi^{2}=1 \cdot 22\right)$.

The effect of a previous myocardial infarction could be accurately assessed only in elective and acute cases; 43 patients had had a myocardial infarction at some time before aneurysmectomy. Twentysix of these were routine cases, and their operative mortality was $15.4 \%$, whereas the operative mortality in the remaining 196 routine cases with no history of a previous myocardial infarction was $7 \cdot 1 \%$. Although the death rate in the patients with a previous infarct was therefore twice that of those without infarcts, this difference was not statistically significant $\left(\chi^{2}=1 \cdot 13\right)$. Seventeen acute cases had had a myocardial infarct, and their operative mortality was $17.6 \%$ compared with $20 \%$ in the 55 patients with no previous infarct.

The effect of preoperative hypotension has been evaluated in the ruptured cases. We have defined hypotension as a recorded systolic blood pressure under $100 \mathrm{~mm} \mathrm{Hg}$ on admission. The operative mortality was $44 \%$ in the 75 patients in this category and was not significantly different from the $41.5 \%$ in the 94 patients with a systolic pressure of more than $100 \mathrm{~mm} \mathrm{Hg}\left(\chi^{2}=0.028\right)$.

Many cases in this series had been referred from other hospitals in our region. Seventy-nine cases were referred direct from general practitioners or from central hospitals within a five-mile radius, and their operative mortality was $49.4 \%$, whereas for the 80 cases who travelled from peripheral hospitals the death rate was $40 \%$. This apparent difference is not statistically significant $\left(\chi^{2}=1.05\right)$ but merely suggests that the distance travelled influences case selection.

Thirty-three patients undergoing resections were asymptomatic, and none of these died postoperatively. In addition to the 27 patients not considered for resection a further 22 patients, initially considered unsuitable for elective resection, subsequently presented as emergencies, six as acute cases requiring urgent operation and 16 as emergencies with ruptured aneurysms. Two of the acute cases and eight of the ruptured died postoperatively, giving an operative death rate of $63.6 \%$ in this deferred group.

\section{Five-year survival}

Long-term survival after successful resection was assessed in the 296 patients available for analysis at the end of 1978. Eighteen patients were lost to follow-up, and in 35 the follow-up was incomplete, leaving 243 available for analysis by the life table method. The overall five-year survival of these 243 patients was $64.8 \% ; 68.1 \%$ in 142 elective cases, $57.1 \%$ in 65 ruptured cases, and $66.0 \%$ in the acute cases (fig 1). The five-year expected survival of a similar population with an initial age of 68 is $76.5 \%$ (fig 2). The difference in long-term survival between elective and ruptured cases is not significant in the Mantel Chi-squared test ${ }^{5}\left(\chi^{2}=1 \cdot 70\right)$.

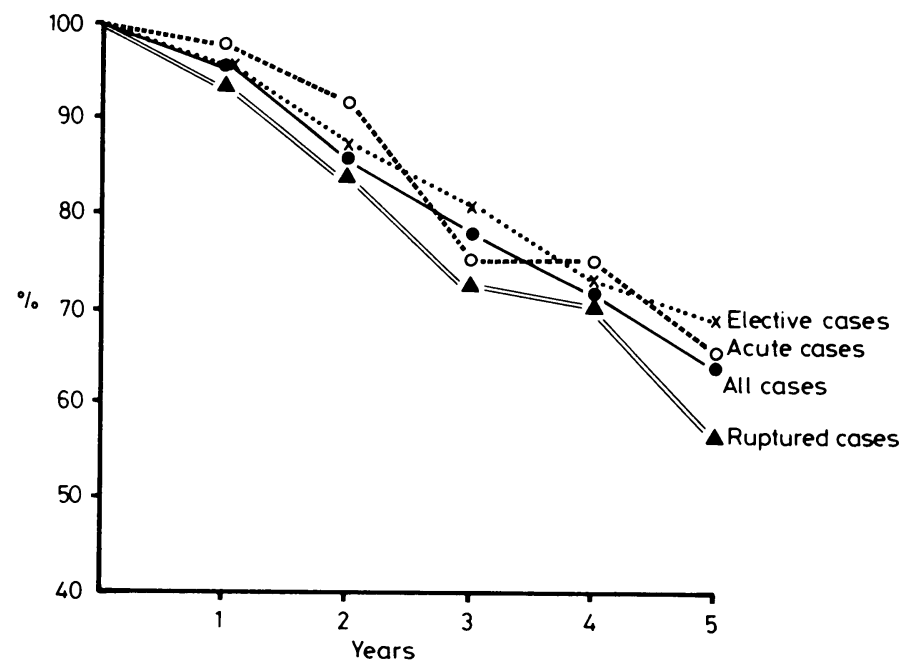

FIG 1-Five-year survival after successful resection of an aneurysm by Lifetable method. ( $\longrightarrow$ all cases, $\mathrm{X}-\cdots \mathrm{X}$ elective cases, $\Delta-\boldsymbol{\Delta}$ ruptured cases, $\bigcirc-\ldots$ acute cases.)

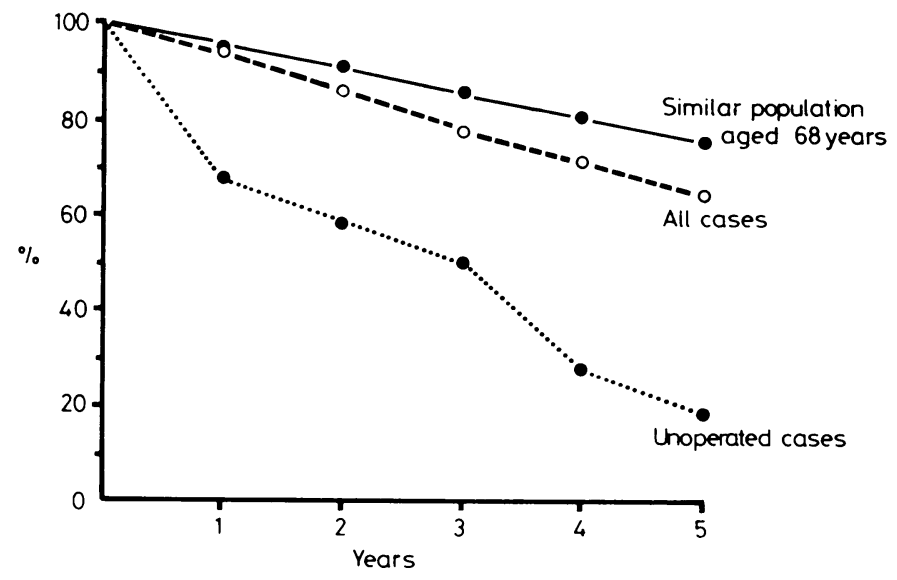

FIG 2-Comparison of the five-year survivals between all cases in the reported series, unoperated cases, ${ }^{6}$ and a similar population aged 68 .

Interestingly during the subsequent follow-up period 14 patients developed malignant disease. Thus in addition to nine with a history of malignant disease and the 11 who had had a malignant condition diagnosed on admission, 34 patients $(6.4 \%)$ in this study have had a malignant neoplasm. 


\section{Discussion}

The replacement of aortic aneurysms has been a major surgical development, and evaluation of experience permits detailed analysis of the results of present management and indicates ways in which results may be further improved by awareness of symptoms and simple clinical examination.

Firstly, it is important to establish the important part that surgery plays, and this may be fully evaluated only by considering its effect on subsequent survival. There can be no debate about the place of surgery for a ruptured aneurysm, when resection and prosthetic implant offer the only hope of the patient's survival. The place of elective surgery may be determined by considering the factors that increase the mortality and morbidity associated with surgery and by assessing the long-term survival of those patients who have undergone resection. Estes ${ }^{\circ}$ studied 102 patients with abdominal aortic aneurysms in a period before replacement surgery was possible and found that the five-year survival rate was $18.9 \%$ (fig 2); moreover, $63.3 \%$ of the deaths were due to rupture of the aneurysm. Szilagyi et $a l^{7}$ in a comparative study of patients treated surgically and non-surgically showed decreased life expectancy in all patients with an aortic aneurysm when compared with a normal population. This deficit was $85 \%$ in patients treated non-surgically and $25 \%$ for surgically treated cases: thus surgery contributed significantly to increased life expectancy. In our series the fiveyear survival by life table was $64 \cdot 8 \%$ for all cases after a successful resection; furthermore, there was no significant difference in long-term survival between those undergoing elective or emergency resection. This is a much better overall five-year survival rate than that for most of the common surgically treated malignancies.

The most important influence on survival is postoperative mortality, which is closely related to the condition of the aneurysm at the time of resection. In our series the overall postoperative mortality associated with ruptured aneurysms was $42.5 \%$, with acute aneurysms $19.4 \%$, and elective aneurysms $8 \cdot 1 \%$. These differences were highly significant statistically, and the operative mortality associated with elective resection compares favourably with that after other major operations, such as colorectal resections for cancer in this age group.

Thus surgery improves the prognosis of patients with abdominal aortic aneurysms, and this could be further improved if a higher proportion of aneurysms were resected electively. Our series, like others, ${ }^{8}$ shows that most patients have symptoms (table IV). Despite this, the diagnosis is unfortunately often missed, as attested by the fact that $32.9 \%$ of the entire series presented as emergencies with ruptured aneurysms: moreover, this incidence has not changed significantly in the past 20 years (table II). The most common presenting symptoms were abdominal and back pain, the latter occurring with increasing frequency when the aneurysm begins to enlarge and is often misinterpreted as "lumbago" or a disc syndrome. The diagnosis can usually be easily established on clinical grounds by the presence of a pulsatile mass, which was present in $91 \%$ of our cases; and the symptomatic aneurysm must always be referred for surgery.

If additional investigations are considered necessary plain, lateral, and posteroanterior abdominal radiography is the most useful in confirming the clinical diagnosis by showing the characteristic curvilinear calcification in the wall of the sac. We do not undertake routine aortography as it is still associated with some morbidity and may even provide misleading information when the aneurysm sac is filled with thrombus, leaving a deceptively normal lumen through it. Aortography, however, does provide information about the distal vessels and the proximal extent of the aneurysm, and should be used when such information is required. Clinically, if one can determine a sulcus between the superior border of the aneurysm and the costal margin the aneurysm will usually prove to be infrarenal. By using these techniques $99 \%$ of infrarenal aortic aneurysms were diagnosed correctly.
Other factors that may influence the decision to proceed to surgical resection are the age of patient, concommitant disease, the size of the aneurysm, and the absence of symptoms. De Bakey et $a l^{9}$ reported that age and co-existent heart disease were influential factors in postoperative mortality, though the apparent differences were not analysed statistically. In our series, while it initially appeared that age, previous myocardial infarction, and the distance travelled in ruptured cases affected postoperative mortality, these apparent differences were not statistically significant. These factors, therefore, should certainly not be considered as contraindications to surgery, but they must obviously be carefully considered in assessing the risk in the individual case.

The size of an aneurysm at first presentation has been considered important, and the place of surgery may be evaluated by comparing the death rates between those treated surgically or conservatively. Survival rates with aneurysms under $6 \mathrm{~cm}$ are better than those with larger aneurysms, but the survival of patients with small untreated aneurysms are significantly worse than those with small surgically treated aneurysms. ${ }^{7} \mathrm{~A}$ small aneurysm does not preclude rupture; indeed, in Szilagyi's series $20 \%$ of the aneurysms of under $6 \mathrm{~cm}$ ruptured. Darling et al ${ }^{10}$ also showed that rupture and death occurred in $9.5 \%$ of patients with an aneurysm of under $4 \mathrm{~cm}$ and in $23 \%$ with a diameter of $4.5 \mathrm{~cm}$. Bernstein et al ${ }^{11}$ using ultrasound have shown that small aneurysms enlarge on average $0.34 \mathrm{~cm}$ a year, though more dramatic changes could also occur. Thus size alone is not a reason for withholding excision.

Some patients with aortic aneurysms have no symptoms. We found $9 \cdot 1 \%$ of all patients and $15 \%$ of patients having an elective resection to be in this category, much lower than in many other series. ${ }^{6} 8$ The decision to recommend surgical resection in these patients should be related to size of the aneurysm and the general condition of the patient. In our series 22 patients. who had had resections deferred for medical reasons or lack of symptoms subsequently presented for emergency surgery, and 10 of these died postoperatively. A further 27 patients were not considered for surgery, and five of these subsequently ruptured. Therefore, of the 49 patients in whom surgery was deferred, 21 subsequently ruptured and a further six presented as acute cases; most important of all, of these 27 patients requiring urgent operation, 15 died.

Conservative management should therefore be considered only in asymptomatic poor-risk patients with an aneurysm of under $6 \mathrm{~cm}$. They should be kept under close observation and modern non-invasive investigations should be used, such as ultrasound or computed tomography; should the aneurysm enlarge or symptoms develop resection should be undertaken forthwith.

We reiterate that, in this series, the overall death rate of elective resection is $8.1 \%$, a figure that, though formidable, compares favourably with that of major abdominal surgery in this age group. Furthermore, if the patient is denied surgery and presents with acute symptoms the operative death rate is more than doubled and if the aneurysm is allowed to rupture the death rate is increased five-fold. Consideration of all these facts suggest that most infrarenal aortic aneurysms may be diagnosed easily by simple palpation of the abdomen, and failure to do this may have disastrous consequences for the patient. Moreover, once the diagnosis is established surgery should be withheld only after most careful consideration of all the relevant factors, and even so a considerable proportion of these patients will ultimately require operation.

In conclusion, we emphasise that surgical treatment should now be the standard management of aneurysms of the abdominal aorta. Life expectancy is increased when compared with conservatively treated cases, and the five-year survival rate after elective aneurysmectomy in our hands is $68 \%$, which compares favourably with that of $76.5 \%$ in a normal population of a similar age. There are few contraindications to elective resection, and further improvements will be achieved only by increasing the number of patients treated electively. This can, and must, 
be achieved by increasing awareness that the condition is common and that the diagnosis can, and should, be made in most cases by careful routine palpation of the abdomen. Our experience, and that of many others, indicates that it is imperative to establish the diagnosis and initial treatment before the onset of acute symptoms or rupture if the death rate of this common disorder is to be appreciably reduced.

We thank all our colleagues but especially those in the department of surgery, our anaesthetists, and nursing staff, who have helped in the management of these patients, and we thank Miss P Cole, Mrs L Whyley, Mrs C Hail, and Mrs J Sharpe for preparing the manuscript and illustrations.

Requests for reprints to: Professor G Slaney.

\section{References}

1 Turk KAD. The post-mortem incidence of abdominal aortic aneurysms. Proc R Soc Med 1965;58:869-70.
2 Bergan JJ, Yao JST. Modern management of abdominal aortic aneurysms. Surg Clin North Am 1974;54:175-93.

${ }^{3} \mathrm{McGregor}$ JC. Unoperated ruptured abdominal aortic aneurysms: a retrospective clinicopathological study over a 10 year period. $\mathrm{Br} \mathcal{F}$ Surg 1976;68:113-6.

${ }^{4}$ Hill AB. Principles of medical statistics. London: Lancet Ltd, 1966.

${ }^{5}$ Mantel N, Haenszel W. Statistical aspects of the analysis of data from retrospective studies of disease. 7 Natl Cancer Inst 1959;22:719-48.

${ }^{6}$ Estes J. Abdominal aortic aneurysms : a study of one hundred and two cases. Circulation 1950;2:258-64.

7 Szilagyi DE, Smith RF, DeRusso FJ, et al. Contribution of abdominal aortic aneurysmectomy to prolongation of life. Ann Surg 1966;164: 678-97.

${ }^{8}$ Gordon-Smith IC, Taylor EW, Nicolaides AN, et al. Management of abdominal aortic aneurysm. Br F Surg 1978;65:834-8.

${ }^{9}$ DeBakey ME, Crawford ES, Cooley DA, et al. Aneurysm of abdominal aorta: analysis of results of graft replacement therapy one to eleven years after operation. Ann Surg 1964;160:622-38.

${ }^{10}$ Darling RC, Messina CR, Brewster DC, Ottinger LW. Autopsy study of unoperated abdominal aortic aneurysms: the case for early resection. Circulation 1977;56, suppl 2:161-4.

${ }^{11}$ Bernstein EF, Dilley RB, Goldberger LE, et al. Growth rates of small abdominal aortic aneurysms. Surgery 1976;80:765-73.

(Accepted 1 fune 1981)

\title{
Contemporary Themes
}

\section{Brain death in Britain as reflected in renal donors}

\author{
BRYAN JENNETT，CATHERINE HESSETT
}

\begin{abstract}
The diagnostic mix of 1228 brain-dead renal donors in Britain was similar to that of 479 cases of brain death recently reported from three neurosurgical units. About half the donors came from non-teaching hospitals without a neurosurgical unit, many of them small and distant from the centre. The different circumstances that preceded brain death were examined-namely, diagnosis and whether the fatal ictus of brain damage occurred when the patient was already in hospital-to explain why donors spend varying times on the ventilator. Head injuries accounted for half the donors, and intracranial haemorrhage for almost a third.

While many potential donors are not made available, the size of the pool has been overestimated, particularly in regard to head injury. Reduction in organ donation since "Panorama" has been very uneven, with some places increasing their yield; this suggests reluctance of doctors to initiate donation rather than relatives withholding permission.
\end{abstract}

\section{Introduction}

Cardiorespiratory function can now be maintained for several days after brain function has irreversibly stopped-that is, after

\footnotetext{
Department of Neurosurgery, Institute of Neurological Sciences, Southern General Hospital, Glasgow G51 4TF

BRYAN JENNETT, MD, FRCS, professor

CATHERINE HESSETT, BSC, MSC, research assistant
}

the occurrence of brain death. Meanwhile, renal transplantation has become a routine procedure that brings undoubted benefits to a high proportion of recipients. Both are now relatively frequent events, with about 4000 cases of brain death and almost 1000 renal transplant operations each year in Britain. Although these two technological developments emerged quite independently they tend to be associated with each other, and it is still sometimes supposed that they can lead to a conflict of interest at the deathbed of some brain-damaged patients.

Brain death emerged in the 1950s with the increasing availability of ventilators for resuscitation and was first formally described in 1959. ${ }^{1}$ There is abundant evidence that once brain death has been confirmed the heart always stops within 14 days (usually much sooner) even though ventilation is maintained. ${ }^{2}$ Discontinuing ventilation in these circumstances is therefore now regarded as good medical practice in order to spare the patient the indignity and the family the distress of a prolonged period of fruitless mechanical "treatment." If alternatives to transplantation emerged, brain death would be just as frequent; doctors would still need to decide in each case when it had become inappropriate to persist with mechanical ventilation because the patient was already dead.

Renal transplantation became a routine procedure some time after brain death had become well recognised, and for some years only a proportion of cadaveric kidneys came from braindead patients. In the West Midlands, for example, oniy $25 \%$ of donors offered in 1969 were receiving ventilation; by 1977 the proportion had risen to $65 \%,{ }^{3}$ and since 1978 all donors have been brain dead. ${ }^{4}$ Relatives are more likely to consent to donation when their family member is receiving ventilation, perhaps because the process of seeking permission and arranging nephrectomy can then be conducted in an unhurried and seemly way. The trust that had been slowly built up over recent years between the public, the donor doctor, and the transplant 